\title{
Ein Wiki-basiertes Dokumentationssystem für das Qualitätsmanagement der Hochschule
}

\author{
Jascha Silbermann, Andrea Schmid
}

\section{Zusammenfassung}

Zum verbesserten Management der umfangreichen Pro-zessdokumentation wurde an der Technischen Hochschule Wildau [FH] im Rahmen des fortlaufenden TQM-Prozesses (TQM - Total Quality Management) ein Dokumentationssystem für das Qualitätsmanagement auf Wiki-Basis aufgebaut. Die Organisation der Prozessdokumentation wurde im Zuge dessen von einem Dateibasierten auf einen Wiki-basierten Ansatz umgestellt. Sämtliche Inhalte wurden in das neu geschaffene TQMWiki migriert. Der Aufbau des TQM-Wiki erstreckte sich über ca. sechs Monate und umfasste die Phasen Vorlauf, Aufbau und Abschluss, die Gesamtarbeitszeit belief sich auf ca. 250 - 300 Stunden. Zur technischen Realisierung des Prozessdokumentationssystems wurde die freie Software DokuWiki verwendet. Das TQM-Wiki ermöglicht die kollaborative Verwaltung und effiziente Nutzung der Qualitätsmanagementdokumentation durch alle Beteiligten. Die Kommunikation über Hochschulprozesse wird transparent und bezieht die Hochschulangehörigen ein. Die Dokumente liegen im HTML-Format vor und können in weitere Standardformate exportiert werden. Der Aufwand für eine Vielzahl häufig durchzuführender Veränderungsprozesse wurde durch den Wiki-basierten Ansatz deutlich reduziert.

\section{Abstract}

The TH Wildau [FH] is the first German Technical University of Applied Sciences to have started a total quality management (TQM) approach based on the norms ISO 9001 and PAS 1037. The university's office for total quality management has over the last nine months implemented a new wiki-based quality management documentation system (QMDS) with the goal of improving the storage, structuring, management and dissemination of the entirety of the university's quality management documentation. This new system is completely web-based and will provide a better user experience not only for university staff and students, but also allow the office for quality management to continually improve the quality management documentation with relative ease. The differences between the formerly used file-based system and the newly constructed wiki-based organization and the work flows in use with both approaches are compared and contrasted. In conclusion the wiki-based approach is found to be the more efficient one, as process complexity can be shown to be greatly reduced across a range of standard tasks.

\section{Einleitung}

\section{Qualitätsmanagement an der TH Wildau [FH]}

Die Technische Hochschule Wildau [FH] hat als erste Hochschule Deutschlands ein Qualitätsmanagementsystem nach den Normen ISO 9001 und PAS 1037 aufgebaut, welches im Sommer 2009 erfolgreich zertifiziert wurde (TH Wildau 2009). Das Qualitätsmanagementsystem strukturiert die Hochschulprozesse und legt Verantwortlichkeiten und Dokumentationsanforderungen fest (TH Wildau 2009: 1.4). Die Prozessdokumentation wurde ursprünglich als Sammlung von ca. 100 Microsoft Word Dokumenten aufgebaut, die als
PDF-Dateien konvertiert und auf der Website der TH Wildau $[\mathrm{FH}]$ veröffentlicht wurden.

Die Verwaltung der Prozessdokumentation obliegt dem Büro für Qualitätsmanagement, einer Stabsstelle der Hochschule. Die Qualitätsmanagementbeauftragte (QMB) der Hochschule ist zuständig für die Aufrechterhaltung, Pflege und Weiterentwicklung des Qualitätsmanagementsystems. Da im Sinne des umfassenden und gelebten Qualitätsmanagements alle Hochschulangehörigen der Organisation in die QM-Prozesse einzubinden sind, berät die QMB die Hochschulbereiche in Qualitätsfragen und koordiniert notwendige Anpassungen des Qualitätsmanagementsystems. 
Die Ziele der Einführung des TQM-Wiki sind eine transparente Kommunikation über Hochschulprozesse auf einer strukturierten und leicht bedienbaren Plattform und die Einbindung einer benutzerfreundlichen Arbeitsplattform für die normgerechte Prozessdokumentation. Alle Hochschulangehörigen können sich einen Überblick über die Dokumentation verschaffen und an der Optimierung der Hochschulabläufe mitarbeiten. Für Prozessverantwortliche dient das TQM-Wiki als Arbeitsplattform, um eigene Prozesse zu verbessern. Für Akkreditierungen und Auditierungen bietet das TQM-Wiki einen übersichtlichen Einblick in die Dokumentation des Qualitätsmanagementsystems der Technischen Hochschule Wildau [FH].

Kontinuierliche Verbesserung und kollaborative Veränderungsprozesse

Qualitätsmanagement-Dokumente der Prozessdokumentation unterliegen kontinuierlichen Verbesserungsprozessen im Sinne der ISO Norm 9001 (ISO 9001: 2008, 8.5). Ändern sich Vorgaben oder die Bedingungen vor Ort, so müssen Prozesse angepasst und die Veränderungen in der Prozessdokumentation reflektiert werden. Dabei ist zu beachten, dass zur Protokollierung der Änderungen alle Versionen eines Dokuments aufbewahrt werden müssen. Ferner sind diese Versionen so zu kennzeichnen, dass auch in Zukunft nachverfolgt werden kann, welche Version zu welchem Zeitpunkt gültig war.

Für gewöhnlich müssen alle Änderungen zwischen mehreren interessierten Parteien wie Verwaltung, Auditoren und Qualitätsmanagementbeauftragten abgestimmt werden, was einer nichtlinearen Kommunikation bedarf und einen hohen koordinativen Aufwand auf Seiten des Qualitätsmanagements verursacht. Der herkömmliche Ansatz besteht darin, Microsoft Word Dokumente per E-Mail zu verschicken, die gespeichert, bearbeitet und wiederum per E-Mail versandt werden. Dies führt schon bei wenigen Beteiligten zu einer großen Anzahl von verschiedenen Versionen eines Dokumentes und damit schnell zu einer nur noch schwer zu überblickenden Menge an Informationen. Da im extremen Fall alle Beteiligten jede Version an alle anderen Beteiligten schicken, wächst die Anzahl an Dokumentenversionen quadratisch mit der Anzahl der beteiligten Personen. Dieses Wachstum ergibt sich zwingend aus dem Datei-basierten Ansatz, kann jedoch durch die Wahl eines Wiki-basierten Ansatzes weitgehend aufgelöst werden, wie in Abbildung 1 dargestellt ist (vgl. Wilson 2008). Datei-basiert werden pro Bearbeitungsschritt bis zu N(N-1) Versionen erzeugt (quadratisches Wachstum), Wiki-basiert jedoch nur N Versionen (lineares Wachstum). $\mathrm{N}$ bezeichnet die Anzahl der Beteiligten.

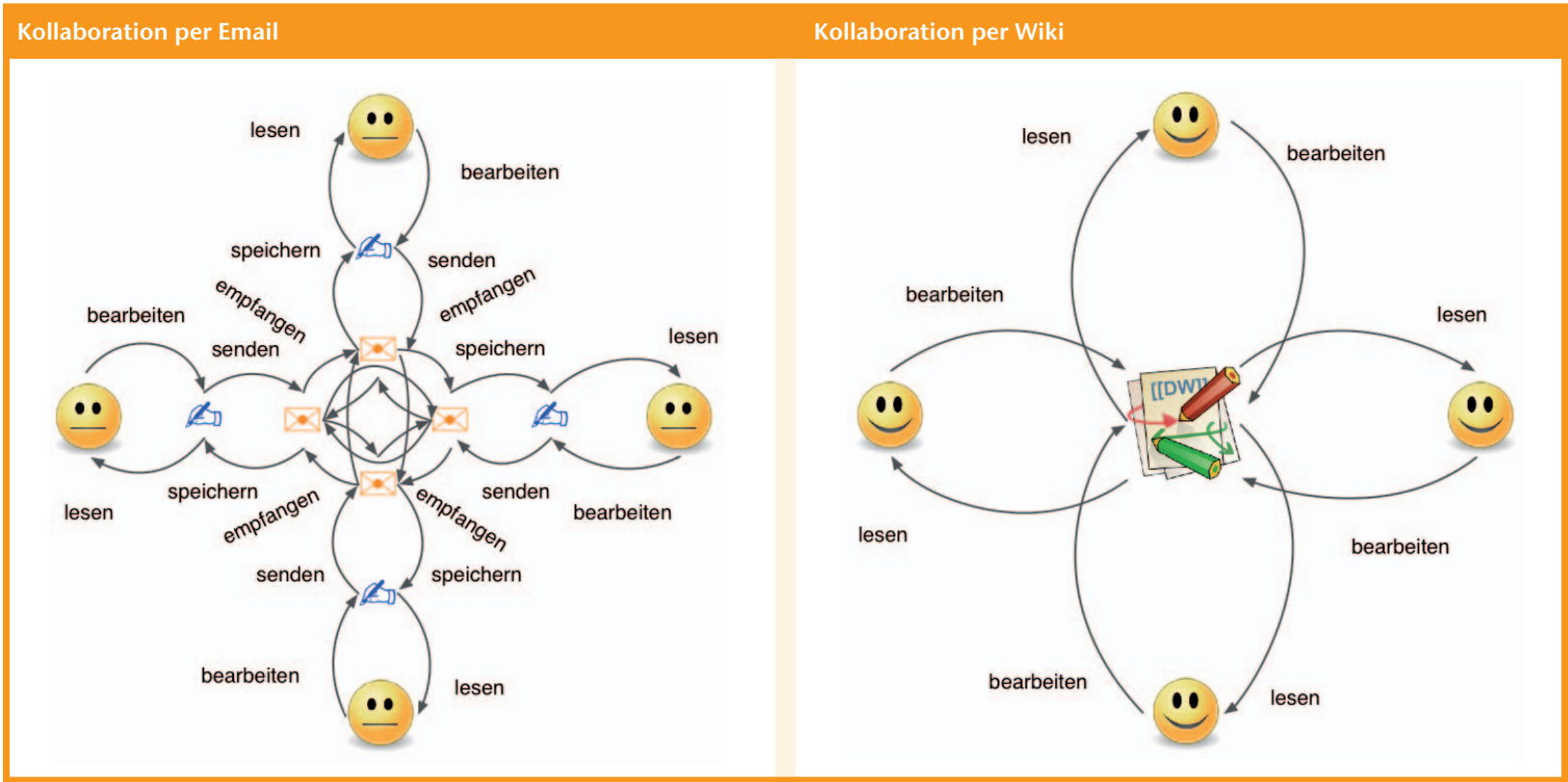

Quellen:

- http://www.wikinomics.com/blog/uploads/frowning_email.jpg

http://commons.wikimedia.org/wiki/File:Face-smile.svg

http://commons.wikimedia.org/wiki/File:Face-plain.svg

http://commons.wikimedia.org/wiki/File:Dokuwiki_logo.svg
Copyright $\odot 2011$ Jascha Silbermann

CreativeCommons by-sa

http://creativecommons.org/licenses/by-sa/3.0/

Abb. 1: Dateibasierter und Wikibasierter kollaborativer Veränderungsprozess 
Wiki- und webbasierte Systeme

Wiki ist ein Ansatz zum kollaborativen Arbeiten mit Informationen, welcher zuerst von Ward Cunningham beschrieben wurde. Cunningham et al. (2002) identifizieren als charakteristische Eigenschaften von Wiki, dass

- die Inhalte in einem speziellen Textformat, der Wiki Syntax, vorliegen,

- an den Inhalten webbasiert kollaborativ gearbeitet werden kann (s.u.),

- Inhalte leicht neu erstellt und untereinander verknüpft werden können.

Neben den eigentlichen Inhalten gelten diese zentralen Punkte auch für die Organisationsstruktur der Inhalte und die Dokumentation der Struktur. Wikis sind damit selbstdokumentierende Systeme. Ein modernes Wiki legt bei jeder Änderung von Inhalten automatisch eine neue Revision an. Änderungen an Inhalten sind somit für die Zukunft dokumentiert. Ferner erlaubt die automatische Versionierung das Rückgängigmachen ungewollter Änderungen.

Um das Wiki-Konzept einzusetzen, bedarf es einer speziellen Software, der sogenannten Wiki-Engine. Diese wird auf einem Standard-Webserver (z. B. Apache) installiert und stellt damit die Wiki-Funktionalitäten unter einer Webadresse zur Verfügung. Auf Basis der Wiki-Engine wird dann das jeweilige Wiki für die Organisation implementiert, in unserem Fall das TQM-Wiki. Die Implementierung umfasst den Aufbau von Strukturen, z. B. von Bereichen im Wiki und das Einpflegen der Inhalte.

Web-basierte Systeme eignen sich hervorragend für den Einsatz in heterogenen Systemen, wie sie in akademischen Einrichtungen anzutreffen sind (Silbermann 2010). Im Gegensatz zum traditionellen Client-Server-Modell, bei der Klienten Software auf den Anwenderrechnern installiert wird, benötigen Web-basierte Systeme nur einen Browser für die interaktive Kommunikation zwischen Anwender und Server. Web-basierte Systeme bieten die folgenden Vorteile:

- Es ist keine Installation spezieller Software auf Seite der Anwender notwendig.

- Die Benutzer interagieren mit dem System durch den ihnen vertrauten Browser.

- Alle Inhalte werden über eine URL angesprochen; sie erhalten dadurch eine Identität und können untereinander verlinkt werden.

\section{Das TQM-Wiki}

\section{Organisationsstruktur der Inhalte}

Das TQM-Wiki beinhaltet die gesamte prozessspezifische Dokumentation der Technischen Hochschule Wildau [FH]. Dazu zählen insbesondere das Hochschulhandbuch mit den Prozessdokumentationen, welches über 60 Abschnitte umfasst. Hinzu kommen rund 30 Prozessbeschreibungen (PB) und Prozesschecklisten (PCL) sowie weitere 30 Flowcharts (Flc) und Formulare (Fbl, vormals »Formblätter «).

Jedes der genannten Dokumente hat im TQM-Wiki einen festgelegten Ort, der sich eindeutig aus dem internen Namen des Dokuments erschließt. So befindet sich beispielsweise der Artikel 1.4 der Prozessdokumentation im Wiki unter »:prozessdoku:1.4«, die Prozessbeschreibung PB 3.1.1 unter »:prozessdoku:pb:3.1.1«. Diese kurzen Pfade bilden also ein Dokument im Wiki eindeutig ab und werden benutzt, um Wikiseiten untereinander zu verknüpfen.

Neben der prozessspezifischen Dokumentation enthält das TQM-Wiki Seiten zu häufig referenzierten Dokumenten der ergebnisspezifischen und externen Dokumentation. Diese Seiten enthalten für gewöhnlich nicht den gesamten Inhalt der Dokumente, sondern weiterführende Informationen, insbesondere Weblinks zu Inhalten auf externen Websites. Dieser Ansatz ermöglicht die konsistente Verknüpfung externer Dokumente und erlaubt, bei Bedarf (z. B. Veränderung der URL eines externen Dokuments) die Änderung nur einmal vorzunehmen, unabhängig davon, wie oft auf das Dokument verwiesen wird. Das bedeutet, dass der Aufwand für die Prüfung des Dokuments auf Änderungsbedarf von linear (jedes Dokument muss überprüft werden) zu konstant (gleicher Aufwand unabhängig vom Umfang der Dokumentation) verringert wird.

Es ist Konvention im TQM-Wiki, dass jedes Dokument, welches ein eigenes internes Kürzel hat - wie z. B. PB 1.4, BbgHG - mit dem internen Kürzel und einem benutzerfreundlichen Titel dargestellt wird. Tabelle 1 gibt eine Übersicht der Inhalte des Wiki mit Pfadangabe und Beschreibung. 


\begin{tabular}{|c|c|c|}
\hline $\begin{array}{l}\text { Bereich } \\
\text { (Struktur) }\end{array}$ & Inhalt & Beschreibung \\
\hline :handbuch & $\begin{array}{l}\text { Hochschulhand- } \\
\text { buch }\end{array}$ & $\begin{array}{l}\text { Das Hochschulhandbuch } \\
\text { enthält die Beschreibung des } \\
\text { Qualitätsmanagementsystems } \\
\text { der Hochschule und legt die } \\
\text { Struktur der Prozessdokumen- } \\
\text { tation fest. }\end{array}$ \\
\hline :prozessdoku & $\begin{array}{l}\text { Prozess- } \\
\text { spezifische } \\
\text { Dokumentation }\end{array}$ & $\begin{array}{l}\text { Die prozessspezifische } \\
\text { Dokumentation umfasst die } \\
\text { Prozessbeschreibungen und } \\
\text { Prozesschecklisten sowie } \\
\text { Flowcharts und Formulare. }\end{array}$ \\
\hline :th & Aufzeichnungen & $\begin{array}{l}\text { Ergebnisspezifische Doku- } \\
\text { mentation wie Studien- und } \\
\text { Prüfungsordnungen und } \\
\text { Amtliche Mitteilungen. }\end{array}$ \\
\hline :codex & $\begin{array}{l}\text { Sammlung von } \\
\text { Normen und } \\
\text { Gesetzen, } \\
\text { externe } \\
\text { Dokumentation }\end{array}$ & $\begin{array}{l}\text { ISO } 9001 \text {, PAS 1037, Hoch- } \\
\text { schulrahmengesetz, Branden- } \\
\text { burgisches Hochschulgesetz } \\
\text { etc. }\end{array}$ \\
\hline :extern & $\begin{array}{l}\text { Externe } \\
\text { Behörden, } \\
\text { Partner und } \\
\text { Kunden }\end{array}$ & $\begin{array}{l}\text { Ministerium für Wissenschaft, } \\
\text { Forschung und Kultur, } \\
\text { Kultusministerkonferenz, } \\
\text { Akkreditierungsrat, Deutsche } \\
\text { Gesellschaft für Qualität etc. }\end{array}$ \\
\hline :datei & Dateien & $\begin{array}{l}\text { Abbildungen und Flowcharts, } \\
\text { die im Wiki eingebunden sind. }\end{array}$ \\
\hline :entwurf & Entwürfe & $\begin{array}{l}\text { Entwürfe der prozessspezifi- } \\
\text { schen Dokumentation. }\end{array}$ \\
\hline :tqm & $\begin{array}{l}\text { Büro für } \\
\text { Qualitäts- } \\
\text { management }\end{array}$ & $\begin{array}{l}\text { Interner Bereich, umfasst } \\
\text { die Wiki-Administration, } \\
\text { Verbesserungsprojekte und } \\
\text { Publikationen. }\end{array}$ \\
\hline :wiki & $\begin{array}{l}\text { Mehr über das } \\
\text { Wiki }\end{array}$ & $\begin{array}{l}\text { Meta-Seiten wie Index und } \\
\text { Hilfe. }\end{array}$ \\
\hline
\end{tabular}

Tab. 1: Übersicht der Inhalte und deren Struktur im TQM-Wiki (Auswahl)

\section{Revisionen und Zugriffsrechte}

Alle Wikiseiten unterliegen der automatischen Revisionskontrolle. Das bedeutet, dass von jedem Dokument bei einer Änderung durch einen Benutzer eine neue Version angelegt wird. Alle Änderungen werden verfolgt und lassen sich bei Bedarf leicht rückgängig machen. Zum einen nimmt dieses Verfahren neuen Benutzern die Angst, Inhalte bei unsachgemäßer Nutzung zu zerstören, zum anderen wird so die Versionierung der QM-Dokumente realisiert. Die Unterschiede zwischen zwei Revisionen lassen sich auf Knopfdruck anzeigen. Das macht ein aufwändiges manuelles Vergleichen zweier Versionen eines Dokumentes unnötig.
Um die QM-Dokumente vor ungewollten und unautorisierten Zugriffen zu schützen, wurden Zugriffsrechte festgelegt, wie in Tabelle 2 dargestellt (Schreibrechte schließen Leserechte mit ein).

\begin{tabular}{|c|c|c|c|}
\hline & Rechte & & \\
\hline Bereich & Manager & Mitwirkende & Anwender \\
\hline :prozessdoku & $\begin{array}{l}\text { Lesen } \\
\text { (Schreiben } \\
\text { nur QMB) }\end{array}$ & Lesen & Lesen \\
\hline :datei & Schreiben & Lesen & Lesen \\
\hline :entwurf & Schreiben & Lesen & Kein Zugriff \\
\hline :tqm & Schreiben & Kein Zugriff & Kein Zugriff \\
\hline
\end{tabular}

Tab. 2: Zugriffsrechte der Benutzer auf ausgewählte Bereiche

Durch die abgestufte Rechtevergabe ist sichergestellt, dass der Nutzerkreis der Wiki-Inhalte möglichst groß ist, während der Kreis derjenigen Benutzer mit Schreibrechten auf das Minimum beschränkt wird. Die offiziellen Dokumente der Prozessdokumentation können nur mit Authentifizierung durch die Qualitätsmanagementbeauftragte verändert werden. Das Datum der letzten Änderung und der Vermerk, durch wen die Änderung erfolgte, werden auf jedem Dokument angezeigt.

Tabelle 3 schlüsselt die Rollen im TQM-Wiki nach Benutzergruppen auf. Die Inhalte des TQM-Wiki werden vom Büro für Qualitätsmanagement verwaltet. An den Änderungsprozessen können weitere Personen aus der Hochschulverwaltung und Externe, z. B. Auditoren, mitwirken. Die Anwender des TQM-Wiki sind sämtliche Mitglieder der TH Wildau [FH], also Studierende, Lehrkräfte und Verwaltungspersonal. Der Wiki-Admin hat Lese- und Schreibrechte auf alle Bereiche des Wiki und agiert auf Weisung der Qualitätsmanagementbeauftragten.

\begin{tabular}{|l|l|}
\hline Rolle & $\begin{array}{l}\text { Benutzergruppe } \\
\text { Admin }\end{array}$ \\
$\begin{array}{l}\text { Wiki-Admin } \\
\text { (von QMB beauftragt) }\end{array}$ \\
\hline Manager & TQM-Büro: QMB und TQM-Mitarbeiter \\
\hline Mitwirkende & Verwaltung und Externe \\
\hline Anwender & Mitglieder der TH Wildau [FH] \\
\hline
\end{tabular}

Tab. 3: Rollen und Benutzergruppen im TQM-Wiki 


\section{Aufbau des TQM-Wiki}

Das TQM-Wiki wurde auf einem vom Hochschulrechenzentrum der TH Wildau [FH] bereitgestellten Server installiert (Apache Webserver auf CentOS). Dieser Server ist aus dem hochschulinternen Netz unter der Adresse http://tqm.th-wildau.de zu erreichen, jedoch vom Internet abgeschottet. Somit ist die hochschulinterne Prozessdokumentation vor unberechtigten Zugriffen weitgehend geschützt, ohne dass sämtlichen Benutzern ein eigener Zugang eingerichtet werden muss.

Zur technischen Realisierung wurde die Wiki-Engine DokuWiki in der Version 2010-11-07a »Anteater « verwendet (Gohr 2011). Diese zeichnet sich durch eine hohe Flexibilität sowie eine einfache Bedienung und Administration aus und umfasst standardmäßig die bisher dargestellten Features für den Aufbau des TQMWiki wie Revisionierung und Zugriffsverwaltung.

Zusätzliche Softwarekomponenten, sogenannte Plugins, wurden ausgewählt und installiert, wie in Tabelle 4 dargestellt ist. Dazu zählen insbesondere die Plugins dw2pdf und bookcreator, mit denen sich PDFDateien aus Wiki-Seiten erstellen lassen, sowie odt, welches eine Wiki-Seite als OpenOffice-Dokument exportiert und discussion, welches eine Kommentarfunktion für Seiten bereitstellt (DokuWiki Plugin Verzeichnis).

\begin{tabular}{|l|l|}
\hline Plugin & Funktion \\
\hline bookcreator & $\begin{array}{l}\text { Mehrere Wiki-Seiten zu einem PDF-»Buch« zusam- } \\
\text { menfassen. }\end{array}$ \\
\hline discussion & $\begin{array}{l}\text { Kommentare auf Wiki-Seiten von Nutzern sammeln } \\
\text { und zentral erfassen. }\end{array}$ \\
\hline dw2pdf & $\begin{array}{l}\text { Eine PDF auf Basis einer beliebigen Wiki-Seite } \\
\text { erstellen. } \\
\text { Das Design der erstellten Dateien spiegelt das Layout } \\
\text { der Druckversion des Hochschulhandbuches wieder } \\
\text { und lässt sich bei Bedarf leicht anpassen. }\end{array}$ \\
\hline odt & $\begin{array}{l}\text { Eine beliebige Wiki-Seite als OpenOffice Dokument } \\
\text { (ODT-Datei) exportieren. } \\
\text { Das Layout der Dateien ist zentral beschrieben und } \\
\text { lässt sich bei Bedarf ändern. }\end{array}$ \\
\hline
\end{tabular}

Tab. 4: Ausgewählte Plugins und deren Funktion

\section{Projektverlauf}

Der zeitliche Ablauf des Aufbaus ist in Abbildung 2 dargestellt. Die Vorlaufphase umfasste die generelle Abschätzung der Machbarkeit sowie die Auswahl und das Testen der benötigten Softwarekomponenten (Plugins).

Die Aufbauphase machte den Großteil der geleisteten Arbeit aus. In dieser Phase wurden sämtliche Inhalte der Prozessdokumentation ins TQM-Wiki überführt. Dazu wurde aus den vorliegenden Dateien im Portable Document Format (PDF) die textuelle Information unter Zuhilfenahme des frei verfügbaren Tools pdf2text (Xpdf) exportiert. Die dabei entstehenden Plain textDateien wurden im Anschluss manuell in Wiki-Seiten

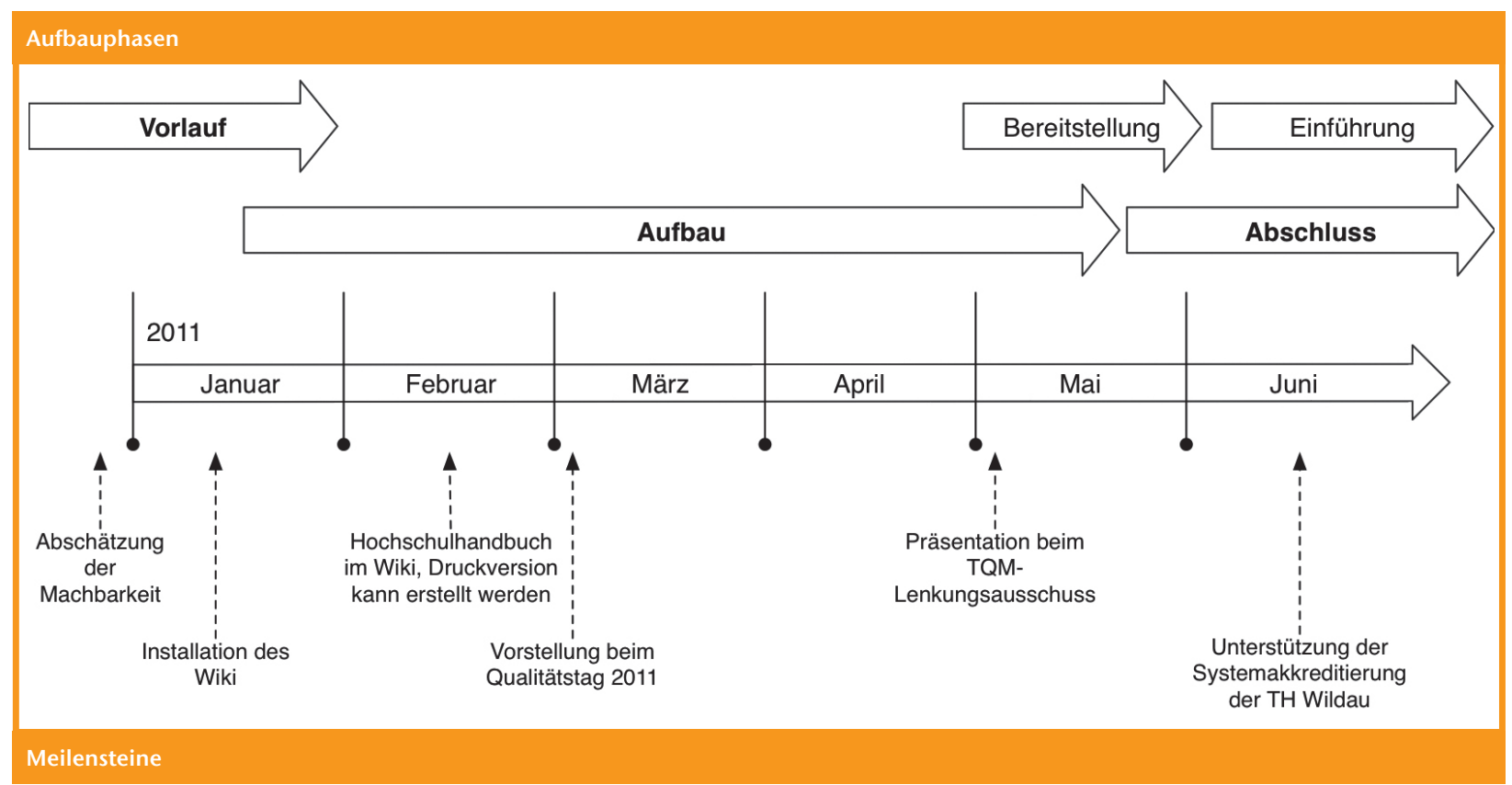

Abb. 2: Aufbau des TQM-Wiki 


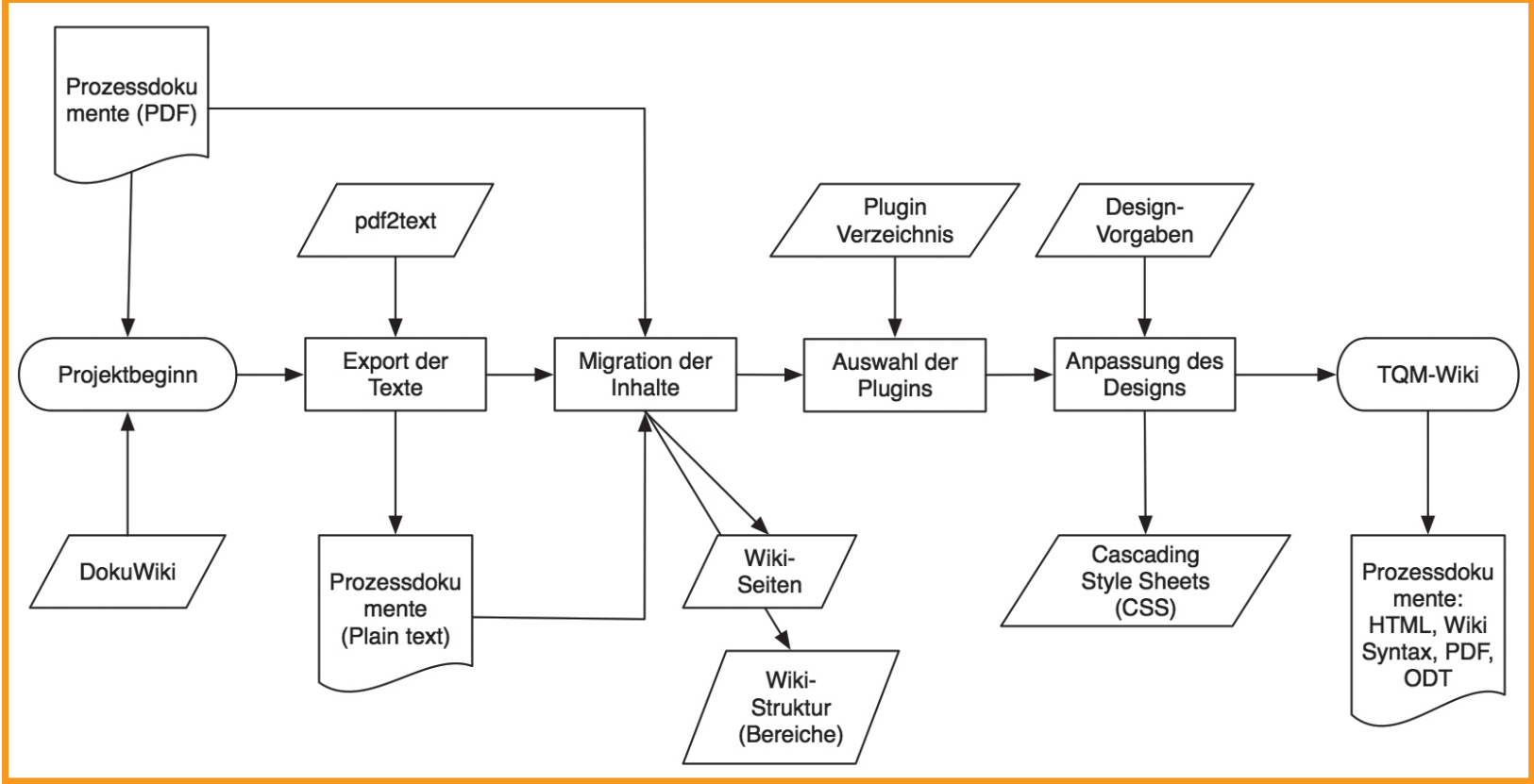

Abb. 3: Migration der Prozessdokumentation

umgewandelt, wobei die Gliederung der Dokumente übertragen wurde. Als visuelle Richtlinie dienten hier$\mathrm{zu}$ wiederum die ursprünglichen PDF-Dateien (siehe Abbildung 3).

Parallel zum Import der Inhalte wurde die zentrale Organisationsstruktur des TQM-Wiki ausgeformt (vgl. Tabelle 1). Die Ausformung der Struktur zielt darauf ab, die Inhalte des Wiki möglichst effizient zu erfassen und diese logisch und leicht nachvollziehbar zu ordnen. Eine gute Strukturierung der Inhalte ist von zentraler Wichtigkeit für die spätere Nutzbarkeit und sollte wohl durchdacht sein, da sich die einmal gewählte Struktur im Nachhinein nur mit erheblichem Aufwand verändern lässt.

Die Abschlussphase umfasste die Veröffentlichung des TQM-Wiki im internen Netz der TH Wildau [FH] und die Bereitstellung des internen Bereichs für die Mitarbeiter des TQM-Büros sowie deren Einarbeitung. Insbesondere schloss dies die Erstellung der Benutzerkonten und der in den Tabellen 2 und 3 dargestellten Rollen und Berechtigungen ein. In einem Pretest wurde das Feedback ausgewählter Nutzergruppen eingeholt. Nach der hochschulweiten Einführung bietet das TQM-Wiki nun die Möglichkeit der kontinuierlichen Rückmeldung der Mitarbeiterinnen und Mitarbeiter zu den Prozessen der Hochschule. Die Abschlussphase erstreckt sich über den Aufbau des TQM-Wiki hinaus und war zum Veröffentlichungszeitpunkt noch nicht abgeschlossen.

\section{Schlussfolgerung}

Mit dem TQM-Wiki wurde eine neue Plattform zum verbesserten Management der Qualitätsdokumentation der TH Wildau [FH] geschaffen. Die Hochschule benutzt nicht als einzige Institution hierfür einen Webbasierten Ansatz. So benutzte Kaewborisut (2005) den Begriff Qualitätsmanagement-Dokumentationssystem für einen Web-basierten Ansatz. Zur Implementierung wurde jedoch keine Wiki-Engine eingesetzt, sondern eine eigene MySQL + PHP-Lösung. Aber auch Wiki-basierte Systeme zur Verwaltung von QM-Dokumentationen sind bereits im Einsatz und werden kommerziell angeboten (Biersack 2009).

Es ist zu erwarten, dass der Einsatz von Wikis in privaten Unternehmen wie in öffentlichen Einrichtungen weiterhin zunehmen wird, da Wikis viele Probleme der traditionellen Datei-basierten Ansätze des Managements von Dokumentensammlungen auflösen. Hierfür sind Wikis insbesondere durch ihre Eigenschaften der automatischen Revisionierung, des Web-basierten kollaborativen Arbeitens und der Verwendung von Standardformaten prädestiniert.

\section{Nutzen, Effizienz und Zukunftsträchtigkeit}

Das TQM-Wiki erlaubt den Mitarbeitern des Büros für Qualitätsmanagement, kollaborativ an Dokumenten zu arbeiten und diese den Anwendern in einem einheitlichen Web-basierten Format zur Verfügung zu 
stellen. Dabei wird eine höhere Effizienz und schnellere Veröffentlichung von Änderungen erreicht, als es vormals möglich war. Die automatische Revisionskontrolle schafft Sicherheit für zukünftige Auditierungen und macht Änderungen nachvollziehbar.

Da nur die QMB die Qualitätsmanagement-Dokumente freigeben kann, wird den Anwendern stets die aktuelle Version eines Dokumentes präsentiert. Anwender können die gesamte Dokumentation durchsuchen, bestimmte Seiten als Lesezeichen im Browser speichern und als OpenOffice- und PDF-Datei exportieren. Ferner können Anwender Kommentare auf Seiten hinterlassen und damit direkt ein Feedback geben und Vorschläge äußern.

Durch die strikte Trennung von Inhalt, Struktur und Darstellung der Wiki-Seiten lässt sich eine sehr viel höhere Zukunftsfähigkeit der QM-Dokumentation erreichen. Die Darstellung der Dokumente sowie der Druck-, OpenOffice- und PDF-Versionen ist separat über Cascading Style Sheets (CSS) definiert und lässt sich bei Bedarf relativ einfach anpassen. Über eine zentrale Änderung kann das Erscheinungsbild aller Dokumente angepasst werden. Das separate Öffnen, Verändern, Abspeichern, Konvertieren, Archivieren und Hochladen sämtlicher Dokumente wird unnötig. Der Aufwand, welcher selbst bei einfachen Änderungen des Layouts, wie dem Einfügen eines neuen Logos, entsteht, wird dadurch von linear auf konstant gesenkt. Tabelle 5 stellt die mit Hilfe des TQM-Wiki erreichte Verringerung des Aufwands von Veränderungsprozessen dar.

\begin{tabular}{|c|c|c|}
\hline Veränderungsprozess & $\begin{array}{l}\text { Aufwand } \\
\text { Datei-basiert }\end{array}$ & Wiki-basiert \\
\hline $\begin{array}{l}\text { Kollaborative Änderung } \\
\text { an Dokumenten }\end{array}$ & $\begin{array}{l}\text { quadratisch: } \\
\text { die Anzahl an } \\
\text { Versionen wächst } \\
\text { quadratisch mit } \\
\text { der Anzahl an } \\
\text { Beteiligten }\end{array}$ & $\begin{array}{l}\text { linear: } \\
\text { die Anzahl an } \\
\text { Versionen wächst } \\
\text { linear mit der } \\
\text { Anzahl der } \\
\text { Beteiligten }\end{array}$ \\
\hline $\begin{array}{l}\text { Prüfung des Änderungs- } \\
\text { bedarfs von Dokumenten } \\
\text { bei Änderungen der } \\
\text { externen Dokumentation }\end{array}$ & $\begin{array}{l}\text { linear: } \\
\text { jedes Dokument } \\
\text { muss einzeln } \\
\text { überprüft } \\
\text { werden }\end{array}$ & $\begin{array}{l}\text { konstant: } \\
\text { die Menge an } \\
\text { Dokumenten mit } \\
\text { Änderungsbedarf } \\
\text { ist bekannt }\end{array}$ \\
\hline $\begin{array}{l}\text { Änderungen am Layout } \\
\text { von Dokumenten }\end{array}$ & $\begin{array}{l}\text { linear: } \\
\text { jedes Dokument } \\
\text { muss einzeln ge- } \\
\text { ändert werden }\end{array}$ & $\begin{array}{l}\text { konstant: } \\
\text { eine Änderung } \\
\text { für alle Doku- } \\
\text { mente }\end{array}$ \\
\hline
\end{tabular}

Tab. 5: Datei- und Wiki-basierter Aufwand von Veränderungsprozessen

\section{Weitere Informationen}

Die Website WikiMatrix enthält Informationen zu über 100 verschiedenen Wiki-Engines und stellt mittels einer ausgeklügelten Vergleichsfunktion die Unterschiede zwischen gewählten Engines übersichtlich dar (WikiMatrix). Weitere Informationen zur Entwicklung des TQM-Wiki finden sich auch online auf den Seiten des Erstautors (Silbermann 2011).

Diese Publikation wurde im TQM-Wiki kollaborativ erstellt und mit den dargestellten Methoden exportiert. Sämtliche Elemente, wie Tabellen, Abbildungen, Textformatierung etc., konnten im Wiki problemlos abgebildet werden.

\section{Literatur}

Biersack, M. (2009): Wikis im Unternehmen: Qualitätsmanagement, Wissensmanagement \& Co., Vortrag, http://www.espresto.de/ media/pdf/Wikis-im-Unternehmen-Qualitaetsmanagement-Wissensmanagement-und-Co_090922.pdf, Zugriff: 28.06.2011.

Cunningham, W., Bo, L. (2002): What Is Wiki?, http://wiki.org/wiki. cgi?WhatIsWiki, Zugriff: 28.06.2011.

DIN EN ISO 9001:2008 (2008): DIN Taschenbuch 226, 6. Auflage, Beuth Verlag, Berlin.

DokuWiki (2011): Plugin Verzeichnis, http://www.dokuwiki.org/ plugins, Zugriff: 28.06.2011.

Gohr, A. (2011): DokuWiki, http://www.splitbrain.org/projects/dokuwiki, Zugriff: 28.06.2011.

Kaewborisut, N. (2005): A web-based application to support quality management documentation system for a construction company. Thesis (M.Eng.), http://library.ait.ac.th/search /a?searchtype=a\& searcharg=Noppadol+Kaewborisut, Zugriff: 28.06.2011.

Silbermann, J. (2011): TQM-Wiki, http://documents.jascha.silbermann.name/reader/?p=tqm-wiki.de, Zugriff: 28.06.2011.

Silbermann, J. (2010): Information Management in the Molecular Biology Lab: Wiki and LIMS. Thesis (M.Sc.), http://documents. jascha.silbermann.name/master-thesis/, Zugriff: 28.06.2011.

TH Wildau (2009): Hochschulhandbuch. http://www.th-wildau.de/ fileadmin/dokumente/hochschule/dokumente/formulare/Hochschulhandbuch_oeffentliche_Version.pdf, Zugriff: 28.06.2011.

WikiMatrix (2011): Compare them all. http://www.wikimatrix.org/, Zugriff: 28.06.2011.

Wilson, M. (2008): Email Versus Wiki Collaboration Graphic a Big Hit. http://wilsonml.wordpress.com/2008/04/08/email-versus-wikicollaboration-graphic-a-big-hit/, Zugriff: 28.06.2011.

Xpdf (2011): A PDF Viewer for X. http://foolabs.com/xpdf/, Zugriff: 28.06.2011. 


\section{Autoren}

Jascha Silbermann,

M.Sc. Biosystemtechnik - Bioinformatik, B.Sc. Bioinformatik

Büro für Qualitätsmanagement, TH Wildau [FH]

Forschungseinheit Molekularbiologie und funktionelle Genomik

Technische Hochschule Wildau [FH]

jascha.silbermann@th-wildau.de

Andrea Schmid, Diplom-Psychologin

Qualitätsmanagementbeauftragte

Leiterin der Stabstelle Qualitätsmanagement der TH Wildau [FH]

Technische Hochschule Wildau [FH]

aschmid@th-wildau.de 